\title{
The orisome: structure and function
}

\author{
Alan C. Leonard* and Julia E. Grimwade \\ Department of Biological Sciences, Florida Institute of Technology, Melbourne, FL, USA
}

During the cell division cycle of all bacteria, DNA-protein complexes termed orisomes trigger the onset of chromosome duplication. Orisome assembly is both staged and stringently regulated to ensure that DNA synthesis begins at a precise time and only once at each origin per cycle. Orisomes comprise multiple copies of the initiator protein DnaA, which oligomerizes after interacting with specifically positioned recognition sites in the unique chromosomal replication origin, oriC. Since DnaA is highly conserved, it is logical to expect that all bacterial orisomes will share fundamental attributes. Indeed, although mechanistic details remain to be determined, all bacterial orisomes are capable of unwinding oriC DNA and assisting with loading of DNA helicase onto the single-strands. However, comparative analysis of oriCs reveals that the arrangement and number of DnaA recognition sites is surprisingly variable among

\section{OPEN ACCESS}

Edited by:

Conrad L. Woldringh,

University of Amsterdam, Netherlands

Reviewed by:

Dhruba Chattoraj,

National Institutes of Health, USA

Elliott Crooke,

Georgetown University Medical

Center, USA

*Correspondence:

Alan C. Leonard

Department of Biological Sciences, Florida Institute of Technology,

LSB 234, 150 West University

Boulevard, Melbourne, FL 32901

aleonard@fit.edu

Specialty section:

This article was submitted to

Microbial Physiology and Metabolism,

a section of the journal

Frontiers in Microbiology

Received: 30 March 2015

Accepted: 18 May 2015

Published: 02 June 2015

Citation:

Leonard AC and Grimwade JE (2015)

The orisome: structure and function.

Front. Microbiol. 6:545.

doi: 10.3389/fmicb.2015.00545 bacterial types, suggesting there are many paths to produce functional orisome complexes. Fundamental questions exist about why these different paths exist and which features of orisomes must be shared among diverse bacterial types. In this review we present the current understanding of orisome assembly and function in Escherichia coli and compare the replication origins among the related members of the Gammaproteobacteria. From this information we propose that the diversity in orisome assembly reflects both the requirement to regulate the conformation of origin DNA as well as to provide an appropriate cell cycle timing mechanism that reflects the lifestyle of the bacteria. We suggest that identification of shared steps in orisome assembly may reveal particularly good targets for new antibiotics.

Keywords: oriC, DnaA, DNA replication, replication origin, DNA binding proteins, orisomes, pre-replication complexes

\section{Introduction}

As the commitment step for proliferation, initiating new rounds of chromosomal DNA synthesis is arguably the paramount event in the life of a bacterial cell. It is also a precarious step, which must rely on sophisticated regulatory mechanisms to ensure that new replication forks are established with sufficient time and number to provide every daughter cell with at least one complete genome copy, regardless of cellular growth rate. Many of these regulatory mechanisms are focused on orisomes, the large multimeric complexes of the bacterial initiator protein, DnaA, that assemble along the unique chromosomal replication origin, oriC.

DnaA is a highly conserved protein whose activity is regulated by the binding and hydrolysis of ATP, with initiation requiring the ATP-bound form (Sekimizu et al., 1987). The crystal structure of a truncated version (domains III and IV) of Aquifex aeolicus has been determined (Erzberger et al., 2002, 2006), revealing that DnaA is not only conserved among bacteria, but also has structural similarity to archaeal and eukaryotic initiatior proteins. Additionally, several 
laboratories have used reverse genetics to introduce targeted mutations in Domains I, III, and IV, and use of these mutants has revealed key roles for these domains in DnaA recruitment (I), binding (IV), oligomerization (I, III), and helicase loading (I, III; see below and representative reviews; Kaguni, 1997; ZawilakPawlik et al., 2005; Mott and Berger, 2007; Ozaki and Katayama, 2009; Leonard and Grimwade, 2011).

Insightful studies on how orisomes assemble, function, and are regulated have come from many laboratories over the course of several decades, mostly using Escherichia coli. Cloning of E. coli oriC onto plasmids (minichromosomes) allowed determination that all instructions for normal orisome assembly are contained in the oriC nucleotide sequence (Leonard and Helmstetter, 1986). Sequencing the cloned oriC (Meijer et al., 1979) identified repeated 9 mer sequences that were determined to be DnaA recognition site (Fuller et al., 1984; Matsui et al., 1985), providing the framework that allowed analysis of how DnaA contacted DNA (Speck et al., 1997; Fujikawa et al., 2003; Yoshida et al., 2003). Seminal studies done by the Kornberg lab provided evidence that initiation could be studied in vitro using crude extracts and purified proteins (Fuller et al., 1981; Kaguni and Kornberg, 1984) and revealed the major stages of orisome assembly (Sekimizu et al., 1988). More recently, high-resolution mapping of DnaA-oriC interactions (McGarry et al., 2004; Rozgaja et al., 2011), biochemical analysis of ordered orisome assembly (Margulies and Kaguni, 1996), and characterization of key subassemblies (Ozaki and Katayama, 2012; Ozaki et al., 2012) have given a clearer picture of how orisomes assemble in E. coli, and, to a lesser extent, in other bacterial types [reviewed in Wolanski et al. (2014)]. However, fundamental questions remain about the relationship between DnaA oligomer formation and the DnaA-directed changes in DNA conformation necessary to unwind the origin, as well as the manner by which orisome assembly is precisely timed in during cell cycle.

The majority of studies on orisomes have been done in vitro, using either DNA fragments or plasmid templates. Plasmids are also useful tools for site-specific mutagenesis, which is essential for dissection of the roles of individual DnaA recognition sites and other oriC sequence elements. However, when the effects of oriC mutations on orisome function were examined in vivo, it became apparent that mutations in cloned oriC do not always have the same effect when placed into the chromosomal context (Weigel et al., 2001). Although the regulatory factors and timing during the cell cycle are identical for plasmid and chromosomal oriCs, there are obvious differences in DNA topology, and intracellular location (Niki and Hiraga, 1999). Most importantly, functional studies of mutant cloned oriCs were usually performed in hosts that harbored wild-type oriC on their chromosomes, setting up a potential competition for available DnaA (Grimwade et al., 2007). Under these conditions the winner ultimately excludes the oriC that loses (does not meet the threshold requirement for DnaA) and causes either death of the host or replacement of the wild-type chromosomal origin with the mutant cloned version. Thus it seems preferable to dissect orisome function in vivo by introducing mutations in chromosomal oriC, but this has, in the past, been a difficult, and labor-intensive process.
The development of $E$. coli recombineering strains that express inducible lambda phage recombination proteins (RED strains; Datsenko and Wanner, 2000; Sharan et al., 2009) has streamlined the introduction of mutations into chromosomal oriC or anywhere else on the genome. Short PCR fragments containing mutant oriC can now be easily inserted as perfect replacements on the chromosome without any additional genetic alterations. Furthermore, these replacements can be performed in strains whose chromosomal replication is under the control of an DnaAindependent, integrated R1 plasmid (Hansen and Yarmolinsky, 1986; Koppes and Nordström, 1986) so that even mutations that render oriC non-functional can be introduced into a DnaA null strain (Kaur et al., 2014). Recently, recombineering strains were constructed with the $s a c B$ gene from Bacillus within oriC to permit selection for oriC replacements on sucrose-containing plates (only strains that lack $s a c B$ will grow; Figure 1). Since the oriC replacement strains still contain an integrated R1 plasmid origin, it is possible to quickly test for function of the mutant oriC by measuring cell viability in the presence of a plasmid that expresses both DnaA and the Rlori repressor (

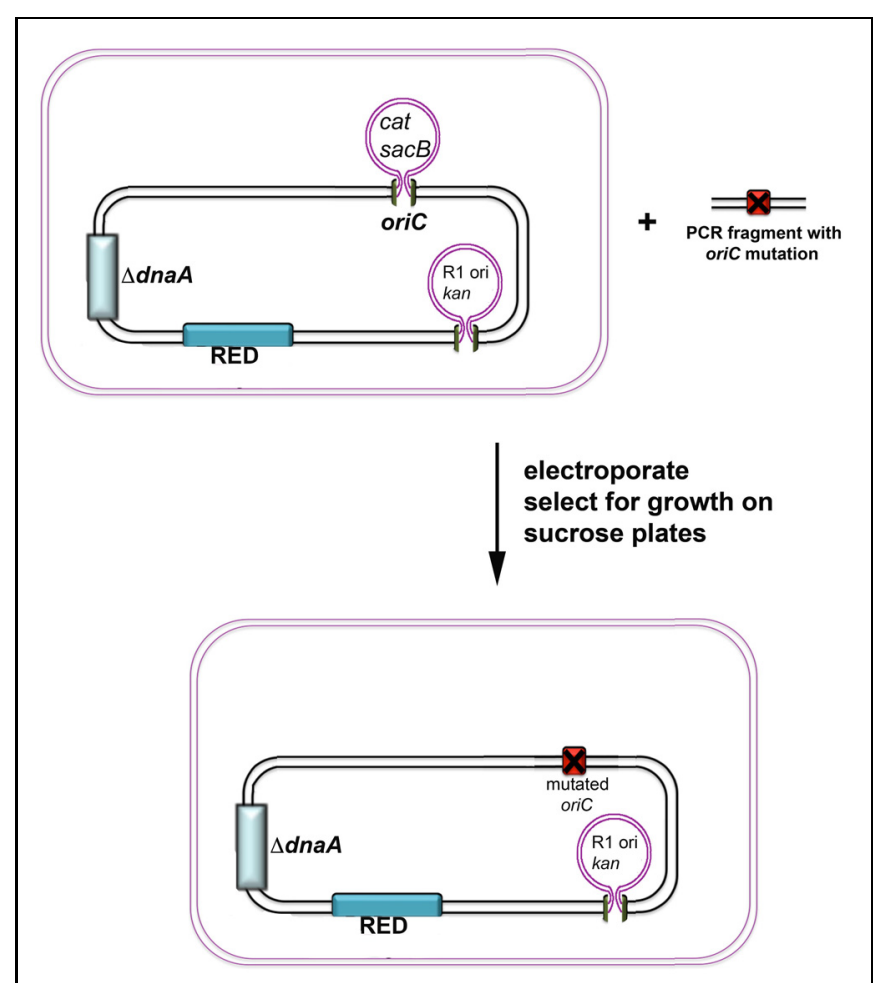

FIGURE 1 | Scheme of the oriC-specific recombineering method. Recombineering strains harbor $\mathrm{sac} B$ and cat genes replacing all of the oriC sequence, inducible genes encoding the lambda RED system, and a plasmid origin of replication (R1ori) linked to a kanamycin resistance determinant. The strain also has a deletion in the dnaA gene. For insertion of oriC mutants into the chromosome, a PCR fragment carrying the desired mutation is electroporated to transform cells in which the RED system was induced. Recombination results in replacement of the sac $B$ and cat genes with the mutated oriC. Successful recombineering confers the ability to grow in the presence of sucrose, and sensitivity to chloramphenicol. 
confirmed by nucleotide sequence analysis, functional oriCs can be transduced into strains with clean genetic backgrounds to study the effect of the mutation on cell growth or cell cycle timing.

Escherichia coli recombineering strains also show promise for the development of novel heterologous systems that will allow in vivo examination of DnaA-oriC interactions that are difficult to perform in native strains, particularly for slow growers or pathogens. Since E. coli DnaA is not required to drive chromosome replication in the recombineering strains, any heterologous oriC and DnaA combination (and any other proteins associated with orisome function) can be introduced at any chromosomal location desired.

\section{Getting Started: The Multifunctional Bacterial Origin Recognition Complex (bORC)}

In order to build orisomes reproducibly during every cell division cycle, there needs to be an invariant starting scaffold that is capable of not only recruiting additional orisome components for later stages of assembly, but also of arranging origin DNA into an appropriate configuration that prohibits DnaA-independent oriC DNA unwinding. This latter feature may be unexpected, but when they are in the supercoiled topology that is required for origin function (Funnell et al., 1986; Von Freiesleben and Rasmussen, 1992), oriC templates contain single-stranded DNA in the absence of any associated protein (Kowalski and Eddy, 1989). Unwinding is observed in an A-T rich region (DNA
Unwinding Element, or DUE; Figure 2A; Kowalski and Eddy, 1989), which is identical to the region which unwinds following orisome assembly (Bramhill and Kornberg, 1988). In E. coli, the DUE contains three 13 mer repeats with consensus sequence 5'-GATCTnTTnTTTT-3', although the nucleotide sequences of DUEs can be quite variable among other bacterial types (ZawilakPawlik et al., 2005).

To avoid premature unwinding, bacterial oriC is usually not naked in cells, but rather is bound to DnaA at high affinity $\left(\mathrm{K}_{\mathrm{d}}\right.$ in the range of 4-20 $\mathrm{nM}$ ) recognition sites (5'-TTATC/ACACA$\left.3^{\prime}\right)$, via a helix-turn-helix (HTH)-type DNA binding motif located within the C-terminal domain (domain IV; Speck et al., 1997; Erzberger et al., 2002; Fujikawa et al., 2003). Three such sites (R1, R2, and R4) are found in E. coli oriC (Figure 2A). DnaA occupies these sites as soon as they becomes accessible, usually after the origin DNA is replicated, and remains bound to them throughout the majority of the cell cycle (Samitt et al., 1989; Nievera et al., 2006). One consequence of this binding is prevention of strand separation, since unwinding of the E. coli DUE, detected by susceptibility to single-strand DNA-specific endonucleases, is completely eliminated when DnaA occupies all three high affinity sites. Endonuclease cutting returns when any single site is unoccupied as a result of mutation (Kaur et al., 2014). This observation is consistent with the idea that supercoiled oriC is constrained by the direct interaction among the bound DnaA molecules. Although the nature of the interaction region is not yet known, a logical candidate is a globular K-homology (KH)-type fold mapped to the N-terminus (domain I; Weigel et al., 1999; Simmons et al., 2003). Domain I is attached to the rest of DnaA by a long flexible linker (domain II; Nozaki and

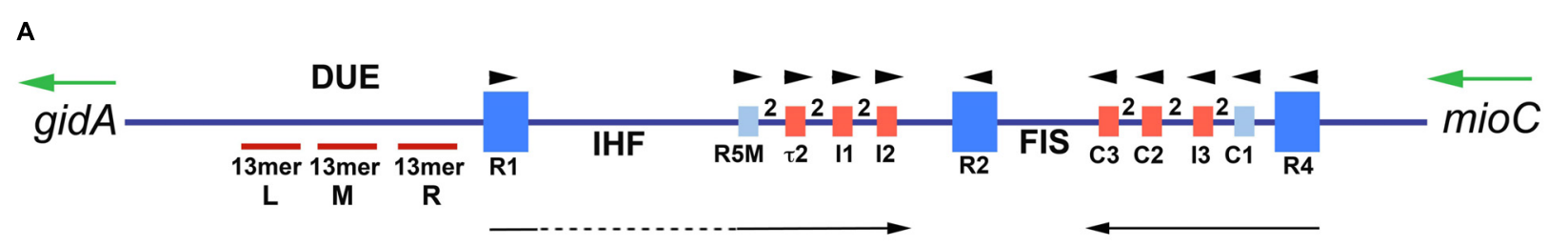

B

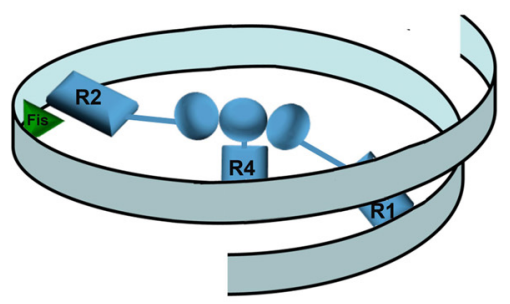

FIGURE 2 | Map of Escherichia coli oriC and conformation of bORC. (A) The oriC region is mapped, showing positions of binding sites for DnaA, IHF, and Fis, as well as the right (R), middle (M), and left $(L)$ 13 mer sequences in the DNA unwinding element (DUE). The three high-affinity sites R1, R2, and R4 are designated by royal blue squares, and the low-affinity sites are marked by small light blue or red rectangles. The red rectangles designate sites that preferentially bind DnaA-ATP, while the sites marked by light blue rectangles bind both nucleotide forms of DnaA equivalently. Small arrowheads show orientation of sites, the two between sites indicates the number of bp separating the sites. Arrows under the map indicate growth direction of DnaA oligomers. The dotted line marks that the oligomer does not span the region between $\mathrm{R} 1$ and R5M. Two genes, gidA and mioC, flanking oriC are shown, with the green arrows marking the direction of transcription. (B) Proposed looped conformation of bORC in E. coli. OriC DNA (ribbon) is constrained by DnaA (gray-blue figures in center of loop) bound at R1, R2, and R4 sites, as labeled. Interaction among the three bound DnaAs proposed to be via domain I. The green triangle represents Fis bound to its cognate site. 
Ogawa, 2008) that could facilitate association of bound DnaA molecules. The high affinity recognition sites in E. coli are widely separated (Figure 2A), and even using the flexible linker, it is difficult to conceive of a way for all the bound DnaA molecules to interact without the formation of DNA loops. An example of a looped bORC is shown in Figure 2B, although this structure is still hypothetical. Since the orisome of $E$. coli appears as a nucleosome-like structure when observed using the electron microscope (Crooke et al., 1993), an intriguing possibility is that as orisome assembly progresses, newly recruited DnaA is added to loosely looped DNA, and then forms oligomers that tighten the loops to form a quasi-nucleosome.

The minimal trimeric DnaA complex bound to E. coli oriC is temporally equivalent to the persistent and well-characterized hexameric eukaryotic origin recognition complex (ORC) that resides at eukaryotic replication origins (Duncker et al., 2009), and we will refer to the bacterial version as bORC. An important difference is that in eukaryotes, ORC is preassembled prior to interacting with replication origins and specific nucleotide motifs are not usually recognized (Bell, 2002; Kawakami and Katayama, 2010). Although DnaA can aggregate in solution, oriC recognition sites are occupied by monomers (Schaper and Messer, 1995; Weigel et al., 1997), with DnaA-DnaA interactions taking place after monomers have bound to DNA.

In additional to being temporally analogous, bORC has functional similarity to the eukaryotic ORC. Like ORC, which recruits additional components of the eukaryotic pre-replication complex, the DnaA in bORC also acts as a scaffold for the recruitment of more copies of DnaA to form subsequent stages of the orisome (Miller et al., 2009; Smits et al., 2011). DnaA molecules are recruited to bORC via domain I (Miller et al., 2009) and a separate DnaA oligomerization region in domain III, encompassing the AAA + (ATPases associated with various cellular activities) fold (Felczak and Kaguni, 2004; Kawakami et al., 2005). Mutations in either domain I or III that abolish DnaA-DnaA interactions also prevent progression of orisome assembly beyond bORC.

Although all three high affinity sites in E. coli oriC are required to constrain the origin, any combination of two high affinity sites are sufficient to assemble orisomes that are functional, albeit with timing defects (Kaur et al., 2014). However, orisomes lacking either R1 or R4 become dependent on two DNA bending proteins that bind to oriC (Fis and IHF, Figure 2 and see below; Kaur et al., 2014), suggesting that there are conformational requirements for bORC. The ability of orisomes to function without DnaA-binding to $\mathrm{R} 1$ also counters previous models that proposed that R1 is essential for DnaA-dependent DNA helicase loading (Speck and Messer, 2001; Soultanas, 2012), and this finding suggests that either R1 is not required for this step, or that another component of the orisome can provide a back-up function when R1 is inactivated.

The nature of bORC in other bacterial types is not well characterized. Clustering of high affinity DnaA recognition sites near A-T rich regions is used as a criteria for mapping bacterial replication origins on newly sequenced genomes (Gao and Zhang, 2007), but the numbers, positions, and orientations of these sites is remarkably variable. Most bacteria carry between 3 and 8 high affinity DnaA recognition sites, but oriC geography is distinctive for each genus (Marczynski and Shapiro, 1993; Zawilak-Pawlik et al., 2005; Gao and Zhang, 2007; Leonard and Mechali, 2013; see also Figure 4). While larger origins usually have higher numbers of high affinity recognition sites, there are, as yet, no hard and fast rules that can be used to predict the configuration of initiator binding sites in oriCs. For example, the thermophile Thermus thermophilus carries a $300 \mathrm{bp}$ oriC with 13 consensus or near consensus recognition sites (Schaper et al., 2000). In Caulobacter, there is only one high affinity DnaA recognition site, but many recognition sites for the regulatory protein CtrA (Marczynski and Shapiro, 1992). This arrangement allows the initiation step to remain DnaA-dependent, but under the control of an additional regulator that ensures the initiation step is restricted to a particular stage of the cell cycle. In some bacteria, for example, Helicobacter (Donczew et al., 2012), Mycoplasma (Cordova et al., 2002; Lartigue et al., 2003), and Bacillus (Moriya et al., 1999), there are two separated clusters of high affinity DnaA recognition sites. This arrangement produces a bipartite configuration whereby DnaA bound at each cluster can interact, but this interaction becomes dependent on DNA bending.

\section{Filling the Gaps: Ordered Orisome Assembly is Determined by Low Affinity DnaA-oriC Interactions}

Since DnaA occupies high affinity sites throughout the cell cycle, mechanisms regulating progression of orisome assembly beyond bORC must be focused on lower affinity DnaA-oriC interactions. Low affinity DnaA recognition sites are mapped in a variety of bacterial oriCs (Charbon and Lobner-Olesen, 2011; Leonard and Mechali, 2013) but these sites are often difficult to identify because they deviate substantially (two or more bases) from the consensus sequence. Thus, direct measurement of DnaA contacts with these sites is often required to confirm their role in orisome assembly (Rozgaja et al., 2011). In E. coli, the recognition sites that are not bound in the bORC have at least a 50-fold lower DnaA binding affinity than do R1, R2, and R4 (Schaper and Messer, 1995). Most importantly, low affinity DnaA recognition sites cannot become occupied unless DnaA is recruited by bound DnaA at a nearby site, and this assistance requires DnaA's domain I (Schaper and Messer, 1995; Rozgaja et al., 2011). For this reason, it is not surprising that the low affinity DnaA recognition sites in E. coli oriC lie in the two DNA gaps (Leonard and Grimwade, 2010) flanked by high affinity recognition sites (see Figure 2A). The arrangement of the low affinity recognition sites within these gaps was unexpected, but provides insight into how cooperative binding leading to DnaA occupation of low affinity sites is achieved. Each of two distinct arrays contains four sites, one in each half of oriC (Figure 2A; R5M, t2, I1, and I2 in the left half and $\mathrm{C} 3, \mathrm{C} 2, \mathrm{I} 3$, and $\mathrm{C} 1$ in the right half; Rozgaja et al., 2011). Notably, each low affinity site within an array is separated from its neighbor by exactly two base pairs, which positions DnaA on the same face of the DNA helix. All the recognition sites in an individual array face in the same direction 
in E. coli, and the arrays in each half of oriC are also oriented in opposite directions relative to one another (Rozgaja et al., 2011; see Figure 2). This arrangement allows a DnaA bound to a high affinity site to assist loading at the proximal low affinity site, with the other sites in the array being filled by progressive cooperative binding between two low affinity sites (Rozgaja et al., 2011).

Spacing between adjacent DnaA recognition sites is critical for these cooperative interactions (Hansen et al., 2007). Since the spacing between high and low affinity sites in oriC varies among bacterial types, and since domain I interactions play a key role in cooperative binding at oriC (Miller et al., 2009), this stringency in spacing implies that the length of the flexible linker region in DnaA's domain II may contribute to the efficiency of orisome assembly. Consistent with this idea, deletions that shorten domain II result in an under-initiation phenotype (Molt et al., 2009). Thus, it is not surprising that the length and amino acid sequence of domain II is the least conserved property of DnaA, and it is likely that among different bacterial types, there is a direct relationship between linker length/flexibility and placement of recognition sites in oriC (Nozaki and Ogawa, 2008).

It should be noted that in some bacterial types, the affinity for DnaA may not be as high as is measured for R1 and R4 in E. coli oriC, and many closely spaced consensus recognition sites may be required for co-operative DnaA binding, as exemplified by the oriC of Thermus (Schaper et al., 2000), and Streptomyces (Jakimowicz et al., 2000). Specific spacing requirements will become more apparent as new low affinity sites are identified in different bacterial origins, and this will necessitate substantial revisions of existing oriC maps, as has happened for $E$. coli (Leonard and Grimwade, 2011). It is also important to not rule out the role of DNA topology in the ability of DnaA to access its recognition sites, and supercoiling dependent DnaA binding in oriC is reported for the oriC of Helicobacter pylori (Donczew et al., 2014).

\section{Dynamic Conformational Switching in oriC: Regulation of Staged Orisome Assembly}

The precise positioning and spacing of DnaA recognition sites encoded into $E$. coli oriC, described above, provides the instructions for ordered orisome assembly. Starting from bORC, the first cooperative binding that should take place, based on proximity, is between DnaA bound at R4 and the adjacent low affinity site C1 (Figure 2A), which nucleates a DnaA oligomer that grows by progressive binding of the low affinity sites in the gap between R4 and R2 (Rozgaja et al., 2011; Figure 3). Given the positions and orientations of DnaA recognition sites in the left half of oriC, assembly of a similar DnaA oligomer in the gap region between $\mathrm{R} 1$ and $\mathrm{R} 2$ was predicted and then detected (Rozgaja et al., 2011). Experimental evidence is consistent with both left and right oligomers growing toward R2 (Rozgaja et al., 2011), and this arrangement means that the DnaA occupying R2 primarily acts to anchor the converging oligomers. Complete loss of DnaA binding at R2 is well tolerated (Weigel et al., 2001) so anchoring of the DnaA oligomers does not appear to be a critical step in orisome assembly. The observation that E. coli oriC retains function in the absence of binding to R1 or R4 implies that the DnaA occupying R2 is capable of nucleating oligomers, and this was shown to be true (Kaur et al., 2014), although R2-bound DnaA does not nucleate as efficiently as the DnaA occupying the peripheral sites, particularly in the right half of oriC.

Despite the appealing symmetry of converging DnaA oligomers, there is an obvious difference in the nucleation of oligomers from DnaA bound at R1 and R4, due to their distance from their proximal sites. Extension of DnaA from R4 is easily accomplished, due to close spacing ( $3 \mathrm{bp}$ ) of R4 and C1 (Rozgaja et al., 2011), but there is a 45 bp gap between $\mathrm{R} 1$ and the nearest low affinity recognition site, R5M, and there is evidence that DnaA oligomers nucleated at R1 do not extend into this region (Rozgaja et al., 2011). Rather, to span this 45 bp space, E. coli oriC DNA is bent to bring $\mathrm{R} 1$ and $\mathrm{R} 5$ into proximity, and the DnaA oligomer that grows by progressive binding of DnaA monomers to the left array of low affinity sites is most likely nucleated by cross-strand DnaA-DnaA interactions via domain I (Figure 3). A DNA bending protein, integration host factor (IHF) recognizes a site placed in the gap between Rland R5M (Polaczek, 1990; Figure 2A) and facilitates the interaction between DnaAs bound at R1 and R5M. IHF binding is not essential for R1 to nucleate a DnaA oligomer or for assembly of functional orisomes (Von Freiesleben et al., 2000; Weigel et al., 2001), presumably because the DNA between R1 and R5M is intrinsically flexible, but loss of IHF binding results in perturbed initiation timing.

In rapidly growing cells, assembly of oligomers nucleated by DnaA bound to R1 and R4 is nearly coincident, and correlates closely with the time that IHF binding to its cognate site in oriC can be detected, and with the time of initiation of DNA synthesis (Cassler et al., 1995). Thus, in vivo, assembly of the right and left DnaA oligomers must be both coordinated and precisely timed. This coupled oligomer assembly appears to be critical, since any loss of precisely ordered orisome assembly causes defects in initiation efficiency or timing (Kaur et al., 2014), especially for bacteria undergoing rapid growth, where new rounds of DNA replication must initiate before previous rounds are completed. In rapidly growing $E$. coli where multiple copies of oriC exist, all origins are activated synchronously to ensure that replication is completed for all chromosomes at the correct time (Skarstad et al., 1986).

To ensure orderly DnaA oligomerization, E. coli uses a DnaAdependent switch mechanism that is built around the successive activity of IHF and another DNA bending protein, Fis (Factor for inversion stimulation), whose recognition site is placed in the gap regions between R2 and C3 (Figure 2A; Filutowicz et al., 1992; Ryan et al., 2004). In rapidly growing E. coli, Fis binds to bORC and places a bend in the constrained bORC loop (Cassler et al., 1995; Figure 3). This bend acts as a long distance inhibitor of IHF binding (Ryan et al., 2004), most likely because it prevents additional bending within the loop (Kaur et al., 2014). Close to the time of initiation, DnaA bound to R4 nucleates assembly of the DnaA oligomer bound to the right array of low affinity sites. Fis is displaced from its site during this process (Ryan et al., 2004; 


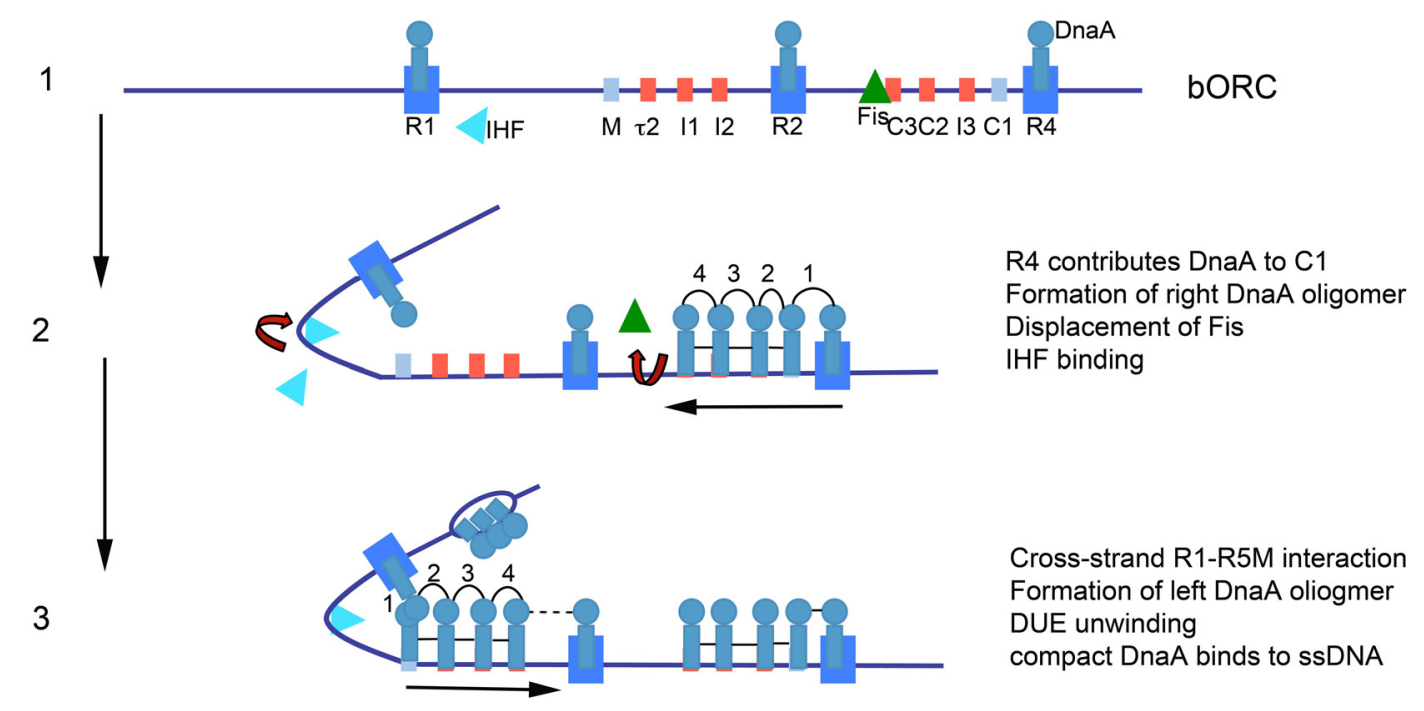

FIGURE 3 | Model of staged orisome assembly. Stage 1 (bORC): after initiation of chromosome replication, DnaA rebinds to high affinity $\mathrm{R} 1, \mathrm{R} 2$, and $\mathrm{R} 4$ sites. Fis is also bound at this stage, but IHF is not. Low affinity sites are unoccupied. Stage 2: DnaA bound to R4 recruits DnaA for binding to C1. DnaA then progressively fills the remaining arrayed sites, forming an oligomer in the gap region between $\mathrm{R} 2$ and $\mathrm{R} 4$. The DnaA oligomer displaces Fis, and loss of
Fis allows IHF to bind to its cognate site. Stage 3: the bend induced by IHF binding allows DnaA, recruited by R1, to bind to R5M, and form a cross-strand DnaA interaction. A DnaA oligomer then progressively grows toward $R 2$, bound to arrayed low affinity sites, and anchored by R2. In this configuration, oriC DNA is unwound in the DUE, and DnaA in the form of a compact filament binds to the ssDNA.
Figure 3), and it is possible that interactions between high affinity sites are also broken, although this has yet to be determined. Displacement of Fis allows IHF to bind and bring DnaA bound to R1 into proximity to R5M (Cassler et al., 1995), resulting in nucleation of the left side oligomer (Ryan et al., 2004; Figure 3). Full occupation of oriC with DnaA is coincident with origin unwinding in the DUE (Grimwade et al., 2000).

The possibility that DNA bending itself may promote unwinding of the DUE was suggested by the timing of the IHF-induced bend. However, in the absence of DnaA, IHF is not capable of creating a single-strand DNA bubble (Grimwade et al., 2000), so some feature of the left oligomer is also required. One possibility is that DNA bending is sufficient for unwinding, but the unwinding is not sustainable, and so the left oligomer is needed to stabilize the unwound single-stranded DNA, and/or participate in DNA helicase loading (Duderstadt et al., 2011; Ozaki and Katayama, 2012). It should be noted that the IHF-induced bend places the DUE in closer proximity to the DnaA bound to the left array of low affinity sites (Figure 3). Alternatively, if bending alone is not sufficient to separate DNA strands in the DUE, then the left oligomer may play a direct role in unwinding, and this has been suggested based on in vitro studies (Ozaki et al., 2012). How left oligomer formation might mediate strand separation is not clearly understood. One model suggests that the DnaA oligomer induces positive supertwists in oriC DNA that result in compensatory negative supercoiling that promotes DNA unwinding of the DUE (Erzberger et al., 2006; Zorman et al., 2012). There is less evidence for a role of the right oligomer in unwinding, since oriC deletion mutants lacking the right half of oriC are viable at slow growth rates, albeit with severely perturbed initiation (Stepankiw et al., 2009). However, the right oligomer cannot be ruled out as a contributor to events in the late stages of orisome assembly, particularly in the loading of DnaB helicase (Ozaki et al., 2012).

The nucleation of DnaA oligomers from high affinity sites is a critical step, and requires domain I-domain I interactions between DnaA molecules (Miller et al., 2009). Given the importance of this stage of orisome assembly, it is logical that it would be a target for regulation. While this remains to be determined, there are several good candidates for regulatory factors. In particular, DiaA (Ishida et al., 2004) and its structural analog HobA (Natrajan et al., 2009; Zawilak-Pawlik et al., 2011), are both positive effectors of orisome assembly, and stimulate the assembly of DnaA oligomers. Both proteins form homotetramers that bind to domain I directly (Keyamura et al., 2009; ZawilakPawlik et al., 2011). E. coli DiaA interacts with a subgroup of DnaA molecules binding to oriC, although the position of these molecules is not known (Keyamura et al., 2007). DnaB helicase and DiaA interact with DnaA domain I at the same location, suggesting that DiaA plays a negative role in the regulation of DNA helicase loading (Keyamura et al., 2009). Although the protein is dispensable, E. coli mutants of DiaA show delayed initiation of chromosome replication (Ishida et al., 2004). HobA is an essential factor in H. pylori (Zawilak-Pawlik et al., 2007), and tetramers of HobA are required for domain I-domain I DnaA oliogmerization (Natrajan et al., 2009). Despite their structural similarity, DiaA and HobA are not interchangeable suggesting a high degree of specificity for the DnaA regulating factors among bacterial types (Zawilak-Pawlik et al., 2011), possibly due to differences in domain $\mathrm{I}$.

Some factors that interact with DnaA domain I are repressors of chromosome replication. This is the case for the E. coli 


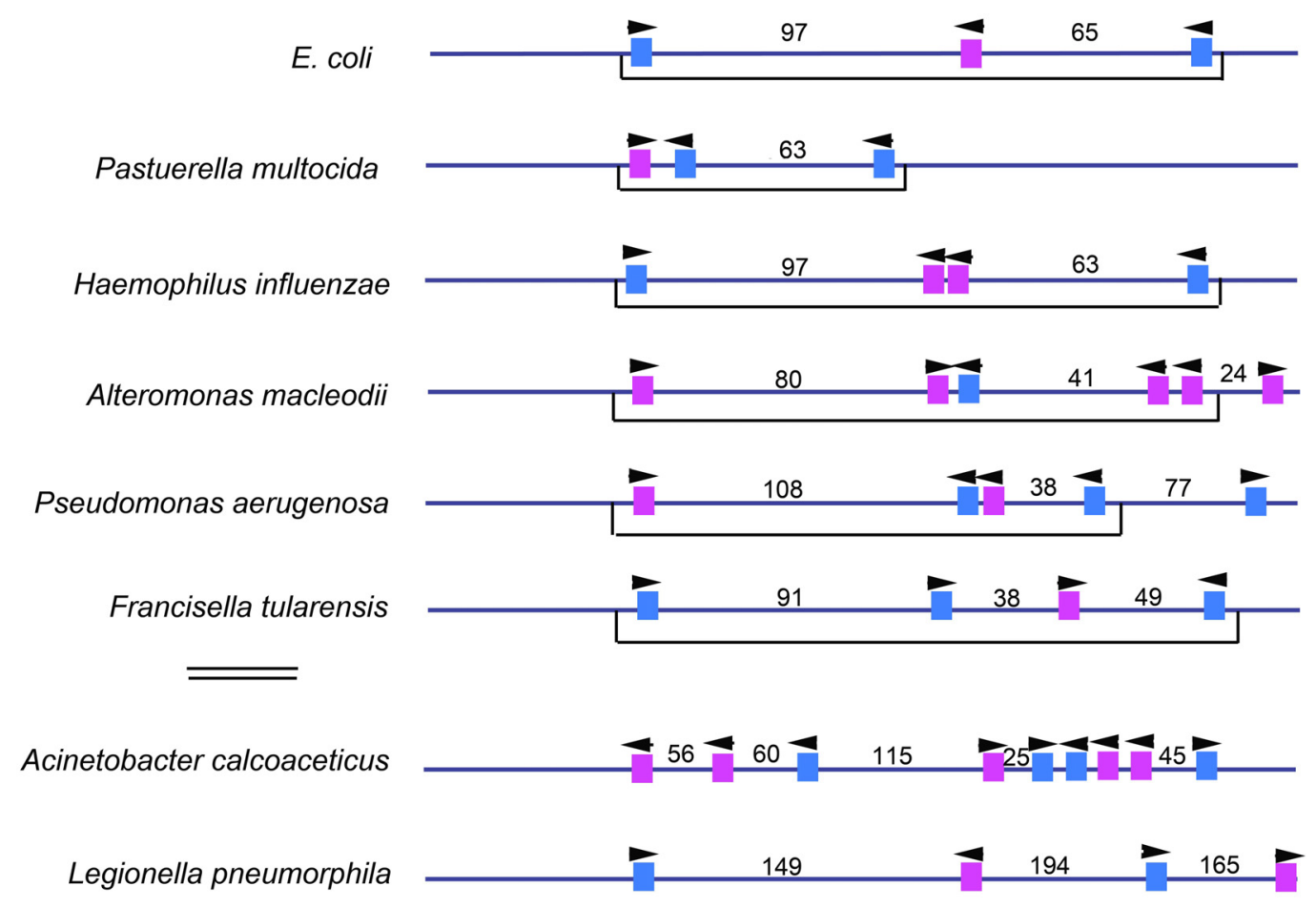

FIGURE 4 | Comparison of the number and placement of high affinity DnaA binding sites in oriCs in related members of the Gammaproteobacteria family. The high affinity sites in the oriC regions of $E$. coli and several related bacterial types are shown. Blue rectangles indicate sites that match the consensus 5'-TTATCCACA, and the pink rectangles mark sites which deviate from this sequence at one or two bases. The arrowheads mark the presumptive orientation of the sites, and the numbers designate the number of base pairs in the gap regions between sites. The brackets below the maps show an E. coli-like arrangement of high affinity sites (see text for details). The two bacterial types below the double line are larger than those above, but it should be noted that the maps are not drawn to scale. ribosomal protein L2 (Chodavarapu et al., 2011), the E. coli starvation protein, Dps (Chodavarapu et al., 2008), and the sporulation-related regulator of Bacillus subtilis, SirA (RahnLee et al., 2011). SirA is an important regulator of ploidy in Bacillus and during sporulation, (Wagner et al., 2009) cells lacking this protein will over-initiate the replication of their chromosomes.

\section{Dissecting the Role of DnaA-ATP in Orisome Assembly and in Timing of Initiation of Chromosome Replication}

New rounds of chromosome replication in most bacteria are dependent on newly synthesized DnaA-ATP and DnaA-ATP is commonly considered to be the "active form" of the initiator. In vitro studies also identified an additional requirement for ATP in the mM range (Sekimizu et al., 1987); this is much higher than is needed for DnaA to bind ATP ( $\mu \mathrm{M}$ range; Sekimizu et al., 1987), and the reason for this requirement has not yet been determined. Additionally, experimental evidence from both in vitro and in vivo studies indicates that some DnaA-ADP is permitted in a functional orisome in E. coli (Yung et al., 1990; McGarry et al., 2004; Grimwade et al., 2007), and it is not yet fully understood what features of orisome assembly and function specifically require DnaA-ATP. One possibility is that DnaA-ATP forms a specific structure on DNA that is required for orisome function. In the absence of DNA, a truncated version (domains III and IV) of Aquifex aeolicus DnaA-ATP assembles into an open ended, compact, right handed, helical filament (Erzberger et al., 2006). In this configuration, an arginine finger in one molecule's domain III contacts the ATP bound to the adjacent protomer. The DNA-binding domain (domain IV) also folds up to contact domain III in the helical filament, implying that a crosstalk mechanism exists between domains III and IV (Duderstadt et al., 2010). These compact DnaAATP filaments can interact directly with single-stranded DNA through a central channel formed by domains III and IV. Two distinct roles, in helicase loading and unwinding, have been proposed for the compact DnaA-ATP filament. Evidence to support these roles comes from in vitro studies, where this structure has been reported to bind the helicase loader, DnaC, and possibly mediate asymmetric loading of the replicative helicase, DnaB (Mott et al., 2008). The filament has also been shown to melt short DNA duplexes, presumably by stretching the helix (Duderstadt et al., 2011). However, the compact filament has very low affinity for double-stranded DNA (Duderstadt et al., 2010), and it is unclear how its assembly could be started in the DUE unless the first protomer was recruited to DNA that was already unwound. 
Although the crystal structure of DnaA-ATP oligomers is compatible with only single-stranded DNA binding, there is also experimental evidence that DnaA-ATP filaments assemble on double-stranded DNA in the gap regions between high affinity site, where they are proposed to mediate unwinding (left gap region) and assist in helicase loading (right gap region; Ozaki and Katayama, 2012). DnaA-ATP oligomers have also been shown to play a key role in Bacillus orisome function in vivo, although it is not known where in oriC these oligomers are formed. Oligomerization of Bacillus DnaA-ATP is stimulated by either single-stranded DNA or double-stranded DNA (Scholefield et al., 2012), but stimulation does not require site-specific DnaA binding and the role of DNA in this reaction remains unclear.

It is also not clear if the oligomers formed on double-stranded DNA must contain only DnaA-ATP. In E. coli, DnaA-ADP and a mutant version of DnaA (R285A), defective in domain III oligomerization, do not fill low affinity binding sites (McGarry et al., 2004; Kawakami et al., 2005), but it is not yet known if this is because only DnaA-ATP can participate in the DnaA-DnaA interactions required for cooperative binding, or because the nucleotide sequence of some of the low affinity 9 mer recognition sites preferentially binds DnaA-ATP (McGarry et al., 2004; Saxena et al., 2011). In support of the latter idea, a single base pair in the 9 mer recognition sequences of I 2 and I 3 is sufficient to convert these sites into ones that bind both nucleotide forms, without increasing their affinity for DnaA-ATP (McGarry et al., 2004). It is not known if low affinity sites that discriminate between DnaA's nucleotide forms are common among different bacterial types.

The structure of the DnaA oligomer associated with doublestranded DNA has not been determined. Duderstadt et al. (2010) suggested that DnaA-ATP forms a less compact oligomer on double-stranded DNA, and single molecule studies examining interaction of DnaA with E. coli oriC are consistent with DnaAATP, but not DnaA-ADP, forming a right handed helical filament on double-stranded DNA (Zorman et al., 2012). However, since E. coli oriC was used as the DNA substrate for the single molecule studies, it is possible the DnaA-ADP did not oligomerize because it was not bound to the origin fragment.

Despite the lack of clarity on how DnaA-ATP participates in orisome function and structure, it is clear that properly timed initiations require tight control of DnaA-ATP binding to oriC. In E. coli, multiple mechanisms, reviewed in Katayama et al. (2010) ensure that the availability of active initiator at oriC fluctuates appropriately during the cell cycle (Kurokawa et al., 1999), and that full occupation of oriC by DnaA is restricted to a short period of time during the cell cycle. These mechanisms, described below, include: (1) converting active DnaA-ATP into the inactive ADPbound form, (2) titrating DnaA by binding it to sites outside oriC, (3) blocking DnaA access to oriC, and when necessary, (4) reactivating DnaA-ADP into active DnaA-ATP.

Mechanisms that inactivate DnaA-ATP activity are often coupled to the elongation phase of DNA replication. In E. coli, conversion of DnaA-ATP to DnaA-ADP is accomplished primarily by interactions with a replisome-associated protein, Hda, which stimulates DnaA's hydrolytic activity in a process termed regulatory inactivation of DnaA (RIDA; Katayama et al., 1998; Katayama and Sekimizu, 1999; Keyamura and Katayama, 2011). Since the nucleotide exchange rate for DnaA in many Gram positives is much higher than in Gram negatives, stimulating DnaA's hydrolysis activity is apparently not an effective regulatory mechanism in all bacteria (Bonilla and Grossman, 2012), and consistent with this idea, Hda is not a conserved protein.

In several bacterial types, DnaA is titrated away from oriC by individual high affinity recognition sites dispersed around the chromosome (Roth and Messer, 1998; Christensen et al., 1999), or by localized clusters of DnaA recognition sites at one, or more positions on the genome. One well-characterized cluster in E. coli, $d a t A$, resides over 450,000 bp away from oriC (Kitagawa et al., 1996; Ogawa et al., 2002) and can titrate large amounts of DnaAATP. At $\operatorname{dat} A$, DnaA assembles into a higher order complex that promotes DnaA-ATP hydrolysis (Kasho and Katayama, 2013). Clusters of DnaA are also found at eight intergenic regions on the Bacillus chromosome (Ishikawa et al., 2007). The conserved YabA protein found in several Gram-positive bacteria may represent a replisome-associated DnaA titration mechanism (Noirot-Gros et al., 2006; Soufo et al., 2008), but YabA is also reported to inhibit cooperative DnaA binding to oriC (Merrikh and Grossman, 2011; Scholefield and Murray, 2013).

Multiple factors in many different bacterial types are reported to bind to oriC and either block DnaA accessibility or affect its cooperative binding/oligomerization. These include CtrA in Caulobacter (Quon et al., 1998), AdpA in Streptomyces (Wolanski et al., 2012), MtrA in Mycobacteria (Rajagopalan et al., 2010), HP1021 in Helicobacter (Donczew et al., 2015), as well as SirA (Rahn-Lee et al., 2011), Soj (Scholefield et al., 2012), and DnaD (Bonilla and Grossman, 2012; Scholefield and Murray, 2013) in Bacillus. The master regulator of Bacillus sporulation, Spo0A, is also reported to bind to oriC, and block rounds of chromosome replication (Boonstra et al., 2013). In E. coli, direct blocking of access to low affinity sites is performed during every cell cycle by the sequestration protein, SeqA (Lu et al., 1994; Slater et al., 1995; Nievera et al., 2006), which recognizes and binds to newly replicated hemimethylated GATC sites that are clustered in oriC and in the $d n a A$ promoter. High affinity recognition sites in oriC are not sequestered and can remain occupied throughout the cell cycle (Nievera et al., 2006), but low affinity oriC recognition sites and transcription of $d n a A$ are blocked for about $1 / 3$ of the cell cycle (Campbell and Kleckner, 1990). It is important to note that mechanisms of these types may play an important role in returning oriC to its correct bORC form, and thereby might turn out to be positive effectors of orisome assembly in addition to repressors.

In rapidly growing $E$. coli, where new rounds of DNA replication are triggered prior to the completion of previous rounds, there is insufficient new of DnaA-ATP synthesis to allow for proper initiation timing at all copies of oriC. A DnaA-ADP recharging system, dependent on two chromosomal loci termed DnaA reactivating sequences (DARSs) raises the levels of DnaAATP, although the exact mechanism remains unclear (Fujimitsu et al., 2009). DARS sites contain a specific arrangement of DnaA recognition sites, which must produce specific interactions 
between bound DnaA-ADP that promotes nucleotide exchange. Interestingly, DARS2 activity is regulated by both Fis and IHF (Kasho et al., 2014), the same DNA bending proteins that regulate orisome assembly. DARS are identified in a number of Gammaproteobacteria and this may be a common regulator for bacteria in this group that are capable of fast growth (Fujimitsu et al., 2009). There is also long standing evidence that DnaA interacts with the acidic phospholipids of the cytoplasmic membrane in E. coli (Crooke, 2001; Regev et al., 2012; Saxena et al., 2013) and like DARS, membranes recharge DnaA-ADP (Garner and Crooke, 1996).

In addition to the multiple mechanisms used to regulate DnaA-ATP levels and oriC accessibility, the existence of sites that preferentially bind DnaA-ATP suggest that the oriC sequence itself is an important component of the cell cycle timing mechanism for chromosome replication in E. coli and any other bacteria with similar sites. To test this idea, several obvious questions must be considered. Is the occupation of DnaA-ATP preferential recognition sites a rate-limiting step for initiation? If so, does each site play an equivalent role in determining the amount of DnaA-ATP needed for initiation, or is accessibility differentially regulated among sites? To answer these questions, it will be necessary to convert each discriminatory site into one that binds both nucleotide forms of DnaA equivalently. From preliminary studies, it appears that such conversion may alter the time of initiation in the cell cycle. For example, changing I 2 and I 3 into non-discriminatory sites on plasmid origins resulted in an origin that was more efficient that the chromosomal oriC, resulting in rapid integration of the cloned version into the host chromosome as a replacement for the wild-type oriC (Grimwade et al., 2007). Further analysis of mutations in individual and combinations of sites will determine their contribution to the cell cycle timing mechanism, but the possibility exists that all of the low affinity DnaA-ATP discriminatory sites in E. coli oriC can be changed into a form that binds DnaA-ADP, while maintaining orisome activity. If this is the case, it would provide strong evidence that that DnaAATP is not required for the assembly of the oligomeric filament along double-stranded DNA or even for initial DNA unwinding, but is required for correct cell cycle timing of initiation. In this scenario, the version of the DnaA-ADP oligomer assembled along oriC would be functionally equivalent to the DnaA-ATP version and it will be necessary to re-evaluate the pervading view of orisome assembly. It is important to note that regardless of the oligomers assembled along the arrayed low affinity sites, DnaA-ATP is also likely to be required later in orisome assembly for single-stranded DNA binding and helicase loading. Further studies should reveal the different roles of DnaA-ATP as a structural component and as a timing feature for the orisome.

\section{Orisomes are an Underexploited Drug Target}

As a component of an essential machine for bacterial growth, and with regulatory proteins that are distinctly different from their eukaryotic counterparts, the orisome might be expected to be an excellent target for antibiotics. However, the orisome, like the replisome, remains under-exploited as a target for new drug development (Robinson et al., 2010). There are no known naturally occurring antibiotics that affect orisomes and perhaps this should be expected for an assemblage comprising a protein that is as highly conserved as DnaA. However, despite the dearth of orisome-specific inhibitors, DnaA activity has been be used as the basis for an antibiotic screen. Robust and high throughput in vivo assays (Fossum et al., 2008) were performed using E. coli conditional lethal, cold-sensitive strains (COS mutants) of DnaA which over-initiate new replication forks (Katayama and Kornberg, 1994). An inhibitor of orisome activity or replication fork movement will restore growth at the nonpermissive temperature (Fossum et al., 2008). The strain also contained an alternative mode of chromosome replication to allow cell survival in the presence of an inhibitor that completely blocked DnaA activity. Using this assay, a novel benzazepinederived, DNA gyrase inhibitor was identified from the Library of Pharmacologically Active Compounds (LOPACs; Johnsen et al., 2010). Although no direct inhibitors of DnaA were identified, over-expression of portions of the E. coli DnaA molecule (domain I or domain IV) were also shown to permit growth at low temperature in a similar assay, presumably by forming inactive hetero-oligomers and blocking orisome assembly (Weigel et al., 1999). Drugs modeled on these domains of DnaA may worth investigating in the future.

Successful orisome inhibitors may be most effective if they are directed toward specific stages or subassemblies of the orisome. Candidate sub-assemblies include the cross-strand DnaA-DnaA structures, promoted by DNA bending proteins like IHF, that may be "Achilles heel" stages that may require more time to assemble, or are less stable than other orisome assembly stages. These stages are likely already to be targets of cellular orisome assembly regulators such as DiaA (Ishida et al., 2004) or HobA (Zawilak-Pawlik et al., 2007).

Another important aspect of targeting inhibitors of orisome assembly is to identify shared steps in orisome assembly among bacterial types. Fortunately, a large oriC database, DoriC, is available for comparative orisome analysis (Gao et al., 2013), but it is obvious from the diverse arrangements of consensus DnaA recognition sites that there are many different ways to assemble orisomes. Furthermore, there is a lack of information on low affinity DnaA recognition sites in bacteria other than E. coli. Although it appears that these sites exist in the well-studied origins of Helicobacter, Mycobacteria, Bacillus, and Caulobacter (Charbon and Lobner-Olesen, 2011; Taylor et al., 2011; Leonard and Mechali, 2013), for comparative analysis, it may be more informative to examine the origins of close relatives (for an example see, Shaheen et al., 2009). The oriC geography for relatives of $E$. coli (members of the Gammaproteobacteria) is shown in Figure 4. Even among the closely related members of this group, the arrangement, number, and orientation of consensus (high affinity) sites is variable. However, a shared, E. coli-like, motif appears with high affinity sites at the boundaries of gap regions (40-100 bp) where low affinity recognition sites would be expected to reside. This motif includes 
E. coli's orientation of high affinity sites and may reflect the common assembly pattern of DnaA oligomers. Interestingly, some members (such as Haemophilus influenza, Pseudomonas aerugenosa, and Alteromonas macleodii) of this group carry extra high affinity sites that are centralized and extremely closely spaced. This arrangement suggests that in these origins, each DnaA oligomer may be anchored at its own high affinity site, rather than sharing one central site (R2) as is the case for E. coli. Other members (such as Acinetobacter calcoaceticus) have multiple high affinity sites downstream or upstream from the shared motif. Although the minimal requirement for high affinity sites remains to be determined for any member of this group other than E. coli (Kaur et al., 2014), additional high affinity sites may be required to build bORCs capable of forming larger or smaller DNA loops or to provide for synthesis of additional DnaA oligomers during staged orisome assembly. Some oriCs in this group (such as $A$. calcoaceticus) may also reflect a bipartite arrangement. Targeting these interesting versions of oriC that deviate from $E$. coli should provide valuable information to set the rules for orisome assembly and identify shared steps for inhibitor targeting.

It is worth noting that the E. coli recombineering strains mentioned above should be useful in developing heterologous systems to map DnaA-oriC interactions in vivo without having to study pathogenic organisms. Any replication origin can be introduced into the $E$. coli chromosome along with appropriate $d n a A$ the gene as replacements for the E. coli versions. The heterologous origin does not need to be functional in these

\section{References}

Bell, S. P. (2002). The origin recognition complex: from simple origins to complex functions. Genes Dev. 16, 659-672. doi: 10.1101/gad.969602

Bonilla, C. Y., and Grossman, A. D. (2012). The primosomal protein DnaD inhibits cooperative DNA binding by the replication initiator DnaA in Bacillus subtilis. J. Bacteriol. 194, 5110-5117. doi: 10.1128/JB.00 958-12

Boonstra, M., de Jong, I. G., Scholefield, G., Murray, H., Kuipers, O. P., and Veening, J. W. (2013). Spo0A regulates chromosome copy number during sporulation by directly binding to the origin of replication in Bacillus subtilis. Mol. Microbiol. 87, 925-938. doi: 10.1111/mmi.12141

Bramhill, D., and Kornberg, A. (1988). Duplex opening by dnaA protein at novel sequences in initiation of replication at the origin of the E. coli chromosome. Cell 52, 743-755. doi: 10.1016/0092-8674(88)90412-6

Campbell, J. L., and Kleckner, N. (1990). E. coli oriC and the dnaA gene promoter are sequestered from dam methyltransferase following the passage of the chromosomal replication fork. Cell 62, 967-979. doi: 10.1016/00928674(90)90271-F

Cassler, M. R., Grimwade, J. E., and Leonard, A. C. (1995). Cell cycle-specific changes in nucleoprotein complexes at a chromosomal replication origin. EMBO J. 14, 5833-5841.

Charbon, G., and Lobner-Olesen, A. (2011). A role for the weak DnaA binding sites in bacterial replication origins. Mol. Microbiol. 82, 272-274. doi: 10.1111/j.13652958.2011.07840.x

Chodavarapu, S., Felczak, M. M., and Kaguni, J. M. (2011). Two forms of ribosomal protein L2 of Escherichia coli that inhibit DnaA in DNA replication. Nucleic Acids Res. 39, 4180-4191. doi: 10.1093/nar/gkq1203

Chodavarapu, S., Gomez, R., Vicente, M., and Kaguni, J. M. (2008). Escherichia coli Dps interacts with DnaA protein to impede initiation: a model of adaptive mutation. Mol. Microbiol. 67, 1331-1346. doi: 10.1111/j.13652958.2008.06127.x strains, since valuable information on orisome assembly can be obtained by examining origin binding to its cognate DnaA and, in the case of non-functional origins, where this process is halted. With a few modifications, a heterologous system of this type should also be useful for orisome inhibitor screening.

In summary, although more challenging experiments await, one can't help but admire the versatility of the instruction set that is encoded into the bacterial origin of replication. Although some of its features are hidden within cryptic nucleotide sequence motifs, these instructions are sufficient to direct the addition of each DnaA subunit to produce staged orisome assembly, as well as encode recognition sites for proteins the produce regulatory switches for orisome assembly as well as binding sites for the regulatory proteins needed for origin resetting. Specific nucleotide sequences are likely to be involved in timing of orisome assembly in the cell cycle and it will be interesting to identify new roles for oriC sequences as different bacterial types are examined and compared. Clearly, despite the intense scrutiny that orisomes have received over the years, they still hold secrets that are worth uncovering.

\section{Acknowledgments}

The work in our laboratories was supported by Public Health Service grant GM54042. Publication of this article was funded in part by the Open Access Subvention Fund and the Florida Tech Libraries.

Christensen, B. B., Atlung, T., and Hansen, F. G. (1999). DnaA boxes are important elements in setting the initiation mass of Escherichia coli. J. Bacteriol. 181, 2683-2688.

Cordova, C. M., Lartigue, C., Sirand-Pugnet, P., Renaudin, J., Cunha, R. A., and Blanchard, A. (2002). Identification of the origin of replication of the Mycoplasma pulmonis chromosome and its use in oriC replicative plasmids. J. Bacteriol. 184, 5426-5435. doi: 10.1128/JB.184.19.5426-5435.2002

Crooke, E. (2001). Escherichia coli DnaA protein-phospholipid interactions: in vitro and in vivo. Biochimie 83, 19-23. doi: 10.1016/S0300-9084(00)0 1224-4

Crooke, E., Thresher, R., Hwang, D. S., Griffith, J., and Kornberg, A. (1993). Replicatively active complexes of DnaA protein and the Escherichia coli chromosomal origin observed in the electron microscope. J. Mol. Biol. 233, 16-24. doi: 10.1006/jmbi.1993.1481

Datsenko, K. A., and Wanner, B. L. (2000). One-step inactivation of chromosomal genes in Escherichia coli K-12 using PCR products. Proc. Natl. Acad. Sci. U.S.A. 97, 6640-6645. doi: 10.1073/pnas.120163297

Donczew, R., Makowski, L., Jaworski, P., Bezulska, M., Nowaczyk, M., ZakrzewskaCzerwińska, J., et al. (2015). The atypical response regulator HP1021 controls formation of the Helicobacter pylori replication initiation complex. Mol. Microbiol. 95, 297-312. doi: 10.1111/mmi.12866

Donczew, R., Mielke, T., Jaworski, P., Zakrzewska-Czerwinska, J., and ZawilakPawlik, A. (2014). Assembly of Helicobacter pylori initiation complex is determined by sequence-specific and topology-sensitive DnaA-oriC interactions. J. Mol. Biol. 426, 2769-2782. doi: 10.1016/j.jmb.2014.05.018

Donczew, R., Weigel, C., Lurz, R., Zakrzewska-Czerwinska, J., and ZawilakPawlik, A. (2012). Helicobacter pylori oriC - the first bipartite origin of chromosome replication in Gram-negative bacteria. Nucleic Acids Res. 40, 9647-9660. doi: 10.1093/nar/gks742

Duderstadt, K. E., Chuang, K., and Berger, J. M. (2011). DNA stretching by bacterial initiators promotes replication origin opening. Nature 478, 209-213. doi: $10.1038 /$ nature 10455 
Duderstadt, K. E., Mott, M. L., Crisona, N. J., Chuang, K., Yang, H., and Berger, J. M. (2010). Origin remodeling and opening in bacteria rely on distinct assembly states of the DnaA initiator. J. Biol. Chem. 285, 28229-28239. doi: 10.1074/jbc.M110.147975

Duncker, B. P., Chesnokov, I. N., and McConkey, B. J. (2009). The origin recognition complex protein family. Genome Biol. 10:214. doi: 10.1186/gb2009-10-3-214

Erzberger, J. P., Mott, M. L., and Berger, J. M. (2006). Structural basis for ATPdependent DnaA assembly and replication-origin remodeling. Nat. Struct. Mol. Biol. 13, 676-683. doi: 10.1038/nsmb1115

Erzberger, J. P., Pirruccello, M. M., and Berger, J. M. (2002). The structure of bacterial DnaA: implications for general mechanisms underlying DNA replication initiation. EMBO J. 21, 4763-4773. doi: 10.1093/emboj/cdf496

Felczak, M. M., and Kaguni, J. M. (2004). The box VII motif of Escherichia coli DnaA protein is required for DnaA oligomerization at the E. coli replication origin. J. Biol. Chem. 279, 51156-51162. doi: 10.1074/jbc.M409695200

Filutowicz, M., Ross, W., Wild, J., and Gourse, R. L. (1992). Involvement of Fis protein in replication of the Escherichia coli chromosome. J. Bacteriol. 174, 398-407.

Fossum, S., De Pascale, G., Weigel, C., Messer, W., Donadio, S., and Skarstad, K. (2008). A robust screen for novel antibiotics: specific knockout of the initiator of bacterial DNA replication. FEMS Microbiol. Lett. 281, 210-214. doi: 10.1111/j.1574-6968.2008.01103.x

Fujikawa, N., Kurumizaka, H., Nureki, O., Terada, T., Shirouzu, M., Katayama, T., et al. (2003). Structural basis of replication origin recognition by the DnaA protein. Nucleic Acids Res. 31, 2077-2086. doi: 10.1093/nar/ gkg309

Fujimitsu, K., Senriuchi, T., and Katayama, T. (2009). Specific genomic sequences of $E$. coli promote replicational initiation by directly reactivating ADP-DnaA. Genes Dev. 23, 1221-1233. doi: 10.1101/gad.1775809

Fuller, R. S., Funnell, B. E., and Kornberg, A. (1984). The dnaA protein complex with the E. coli chromosomal replication origin (oriC) and other DNA sites. Cell 38, 889-900. doi: 10.1016/0092-8674(84)90284-8

Fuller, R. S., Kaguni, J. M., and Kornberg, A. (1981). Enzymatic replication of the origin of the Escherichia coli chromosome. Proc. Natl. Acad. Sci. U.S.A. 78, 7370-7374. doi: 10.1073/pnas.78.12.7370

Funnell, B. E., Baker, T. A., and Kornberg, A. (1986). Complete enzymatic replication of plasmids containing the origin of the Escherichia coli chromosome. J. Biol. Chem. 261, 5616-5624.

Gao, F., Luo, H., and Zhang, C. T. (2013). DoriC 5.0: an updated database of oriC regions in both bacterial and archaeal genomes. Nucleic Acids Res. 41, D90-D93. doi: $10.1093 /$ nar/gks 990

Gao, F., and Zhang, C. T. (2007). DoriC: a database of oriC regions in bacterial genomes. Bioinformatics 23, 1866-1867. doi: 10.1093/bioinformatics/btm255

Garner, J., and Crooke, E. (1996). Membrane regulation of the chromosomal replication activity of $E$. coli DnaA requires a discrete site on the protein. EMBO J. 15, 3477-3485.

Grimwade, J. E., Ryan, V. T., and Leonard, A. C. (2000). IHF redistributes bound initiator protein, DnaA, on supercoiled oriC of Escherichia coli. Mol. Microbiol. 35, 835-844. doi: 10.1046/j.1365-2958.2000.01755.x

Grimwade, J. E., Torgue, J. J., McGarry, K. C., Rozgaja, T., Enloe, S. T., and Leonard, A. C. (2007). Mutational analysis reveals Escherichia coli oriC interacts with both DnaA-ATP and DnaA-ADP during pre-RC assembly. Mol. Microbiol. 66, 428-439. doi: 10.1111/j.1365-2958.2007.05930.x

Hansen, E. B., and Yarmolinsky, M. B. (1986). Host participation in plasmid maintenance: dependence upon dnaA of replicons derived from P1 and F. Proc. Natl. Acad. Sci. U.S.A. 83, 4423-4427. doi: 10.1073/pnas.83.12.4423

Hansen, F. G., Christensen, B. B., and Atlung, T. (2007). Sequence characteristics required for cooperative binding and efficient in vivo titration of the replication initiator protein DnaA in E. coli. J. Mol. Biol. 367, 942-952. doi: 10.1016/j.jmb.2007.01.056

Ishida, T., Akimitsu, N., Kashioka, T., Hatano, M., Kubota, T., Ogata, Y., et al. (2004). DiaA, a novel DnaA-binding protein, ensures the timely initiation of Escherichia coli chromosome replication. J. Biol. Chem. 279, 45546-45555. doi: 10.1074/jbc.M402762200

Ishikawa, S., Ogura, Y., Yoshimura, M., Okumura, H., Cho, E., Kawai, Y., et al. (2007). Distribution of stable DnaA-binding sites on the Bacillus subtilis genome detected using a modified ChIP-chip method. DNA Res. 14, 155-168. doi: $10.1093 /$ dnares/dsm017
Jakimowicz, D., Majkadagger, J., Konopa, G., Wegrzyn, G., Messer, W., Schrempf, H., et al. (2000). Architecture of the Streptomyces lividans DnaA protein-replication origin complexes. J. Mol. Biol. 298, 351-364. doi: 10.1006/jmbi.2000.3686

Johnsen, L., Weigel, C., von Kries, J., Møller, M., and Skarstad, K. (2010). A novel DNA gyrase inhibitor rescues Escherichia coli dnaAcos mutant cells from lethal hyperinitiation. J. Antimicrob. Chemother. 65, 924-930. doi: 10.1093/jac/dkq071

Kaguni, J. M. (1997). Escherichia coli DnaA protein: the replication initiator. Mol. Cells 7, 145-157.

Kaguni, J. M., and Kornberg, A. (1984). Replication initiated at the origin (oriC) of the E. coli chromosome reconstituted with purified enzymes. Cell 38, 183-190. doi: 10.1016/0092-8674(84)90539-7

Kasho, K., Fujimitsu, K., Matoba, T., Oshima, T., and Katayama, T. (2014). Timely binding of IHF and Fis to DARS2 regulates ATP-DnaA production and replication initiation. Nucleic Acids Res. 42, 13134-13149. doi: 10.1093/nar/gku1051

Kasho, K., and Katayama, T. (2013). DnaA binding locus datA promotes DnaAATP hydrolysis to enable cell cycle-coordinated replication initiation. Proc. Natl. Acad. Sci. U.S.A. 110, 936-941. doi: 10.1073/pnas.12120 70110

Katayama, T., and Kornberg, A. (1994). Hyperactive initiation of chromosomal replication in vivo and in vitro by a mutant initiator protein, DnaAcos, of Escherichia coli. J. Biol. Chem. 269, 12698-12703.

Katayama, T., Kubota, T., Kurokawa, K., Crooke, E., and Sekimizu, K. (1998). The initiator function of DnaA protein is negatively regulated by the sliding clamp of the E. coli chromosomal replicase. Cell 94, 61-71. doi: 10.1016/S00928674(00)81222-2

Katayama, T., Ozaki, S., Keyamura, K., and Fujimitsu, K. (2010). Regulation of the replication cycle: conserved and diverse regulatory systems for DnaA and oriC. Nat. Rev. Microbiol. 8, 163-170. doi: 10.1038/nrmicro2314

Katayama, T., and Sekimizu, K. (1999). Inactivation of Escherichia coli DnaA protein by DNA polymerase III and negative regulations for initiation of chromosomal replication. Biochimie 81, 835-840. doi: 10.1016/S03009084(99)00213-8

Kaur, G., Vora, M. P., Czerwonka, C. A., Rozgaja, T. A., Grimwade, J. E., and Leonard, A. C. (2014). Building the bacterial orisome: high-affinity DnaA recognition plays a role in setting the conformation of oriC DNA. Mol. Microbiol. 91, 1148-1163. doi: 10.1111/mmi.12525

Kawakami, H., and Katayama, T. (2010). DnaA, ORC, and Cdc6: similarity beyond the domains of life and diversity. Biochem. Cell Biol. 88, 49-62. doi: 10.1139/o09-154

Kawakami, H., Keyamura, K., and Katayama, T. (2005). Formation of an ATPDnaA-specific initiation complex requires DnaA Arginine 285, a conserved motif in the AAA+ protein family. J. Biol. Chem. 280, 27420-27430. doi: 10.1074/jbc.M502764200

Keyamura, K., Abe, Y., Higashi, M., Ueda, T., and Katayama, T. (2009). DiaA dynamics are coupled with changes in initial origin complexes leading to helicase loading. J. Biol. Chem. 284, 25038-25050. doi: 10.1074/jbc.M109.002717

Keyamura, K., Fujikawa, N., Ishida, T., Ozaki, S., Su'etsugu, M., Fujimitsu, K., et al. (2007). The interaction of DiaA and DnaA regulates the replication cycle in $E$. coli by directly promoting ATP DnaA-specific initiation complexes. Genes Dev. 21, 2083-2099. doi: 10.1101/gad.1561207

Keyamura, K., and Katayama, T. (2011). DnaA protein DNA-binding domain binds to Hda protein to promote inter-AAA + domain interaction involved in regulatory inactivation of DnaA. J. Biol. Chem. 286, 29336-29346. doi: 10.1074/jbc.M111.233403

Kitagawa, R., Mitsuki, H., Okazaki, T., and Ogawa, T. (1996). A novel DnaA protein-binding site at $94.7 \mathrm{~min}$ on the Escherichia coli chromosome. Mol. Microbiol. 19, 1137-1147. doi: 10.1046/j.1365-2958.1996.453983.x

Koppes, L., and Nordström, K. (1986). Insertion of an R1 plasmid into the origin of replication of the $E$. coli chromosome: random timing of replication of the hybrid chromosome. Cell 44, 117-124. doi: 10.1016/0092-8674(86)90490-3

Kowalski, D., and Eddy, M. J. (1989). The DNA unwinding element: a novel, cisacting component that facilitates opening of the Escherichia coli replication origin. EMBO J. 8, 4335-4344.

Kurokawa, K., Nishida, S., Emoto, A., Sekimizu, K., and Katayama, T. (1999). Replication cycle-coordinated change of the adenine nucleotide-bound 
forms of DnaA protein in Escherichia coli. EMBO J. 18, 6642-6652. doi: 10.1093/emboj/18.23.6642

Lartigue, C., Blanchard, A., Renaudin, J., Thiaucourt, F., and Sirand-Pugnet, P. (2003). Host specificity of mollicutes oriC plasmids: functional analysis of replication origin. Nucleic Acids Res. 31, 6610-6618. doi: 10.1093/nar/gkg848

Leonard, A. C., and Grimwade, J. E. (2010). Regulating DnaA complex assembly: it is time to fill the gaps. Curr. Opin. Microbiol. 13, 766-772. doi: 10.1016/j.mib.2010.10.001

Leonard, A. C., and Grimwade, J. E. (2011). Regulation of DnaA assembly and activity: taking directions from the genome. Annu. Rev. Microbiol. 65, 19-35. doi: 10.1146/annurev-micro-090110-102934

Leonard, A. C., and Helmstetter, C. E. (1986). Cell cycle-specific replication of Escherichia coli minichromosomes. Proc. Natl. Acad. Sci. U.S.A. 83, 5101-5105. doi: $10.1073 /$ pnas.83.14.5101

Leonard, A. C., and Mechali, M. (2013). DNA replication origins. Cold Spring Harb. Perspect. Biol. 5:a010116. doi: 10.1101/cshperspect.a010116

Lu, M., Campbell, J. L., Boye, E., and Kleckner, N. (1994). SeqA: a negative modulator of replication initiation in E. coli. Cell. 77, 413-426. doi: 10.1016/0092-8674(94)90156-2

Marczynski, G. T., and Shapiro, L. (1992). Cell-cycle control of a cloned chromosomal origin of replication from Caulobacter crescentus. J. Mol. Biol. 226, 959-977. doi: 10.1016/0022-2836(92)91045-Q

Marczynski, G. T., and Shapiro, L. (1993). Bacterial chromosome origins of replication. Curr. Opin. Genet. Dev. 3, 775-782. doi: 10.1016/S0959437X(05)80098-X

Margulies, C., and Kaguni, J. M. (1996). Ordered and sequential binding of DnaA protein to oriC, the chromosomal origin of Escherichia coli. J. Biol. Chem. 271, 17035-17040. doi: 10.1074/jbc.271.29.17035

Matsui, M., Oka, A., Takanami, M., Yasuda, S., and Hirota, Y. (1985). Sites of dnaA protein-binding in the replication origin of the Escherichia coli K-12 chromosome. J. Mol. Biol. 184, 529-533. doi: 10.1016/0022-2836(85) 90299-2

McGarry, K. C., Ryan, V. T., Grimwade, J. E., and Leonard, A. C. (2004). Two discriminatory binding sites in the Escherichia coli replication origin are required for DNA strand opening by initiator DnaA-ATP. Proc. Natl. Acad. Sci. U.S.A. 101, 2811-2816. doi: 10.1073/pnas.0400340101

Meijer, M., Beck, E., Hansen, F. G., Bergmans, H. E., Messer, W., von Meyenburg, K., et al. (1979). Nucleotide sequence of the origin of replication of the Escherichia coli K-12 chromosome. Proc. Natl. Acad. Sci. U.S.A. 76, 580-584. doi: $10.1073 /$ pnas.76.2.580

Merrikh, H., and Grossman, A. D. (2011). Control of the replication initiator DnaA by an anti-cooperativity factor. Mol. Microbiol. 82, 434-446. doi: 10.1111/j.1365-2958.2011.07821.x

Miller, D. T., Grimwade, J. E., Betteridge, T., Rozgaja, T., Torgue, J. J., and Leonard, A. C. (2009). Bacterial origin recognition complexes direct assembly of higher-order DnaA oligomeric structures. Proc. Natl. Acad. Sci. U.S.A. 106, 18479-18484. doi: 10.1073/pnas.0909472106

Molt, K. L., Sutera, V. A., Moore, K. K., and Lovett, S. T. (2009). A role for nonessential domain II of initiator protein, DnaA, in replication control. Genetics 183, 39-49. doi: 10.1534/genetics.109.104760

Moriya, S., Imai, Y., Hassan, A. K., and Ogasawara, N. (1999). Regulation of initiation of Bacillus subtilis chromosome replication. Plasmid 41, 17-29. doi: 10.1006/plas.1998.1381

Mott, M. L., and Berger, J. M. (2007). DNA replication initiation: mechanisms and regulation in bacteria. Nat. Rev. Microbiol. 5, 343-354. doi: $10.1038 /$ nrmicro 1640

Mott, M. L., Erzberger, J. P., Coons, M. M., and Berger, J. M. (2008). Structural synergy and molecular crosstalk between bacterial helicase loaders and replication initiators. Cell 135, 623-634. doi: 10.1016/j.cell.2008. 09.058

Natrajan, G., Noirot-Gros, M. F., Zawilak-Pawlik, A., Kapp, U., and Terradot, L. (2009). The structure of a DnaA/HobA complex from Helicobacter pylori provides insight into regulation of DNA replication in bacteria. Proc. Natl. Acad. Sci. U.S.A. 106, 21115-21120. doi: 10.1073/pnas.0908966106

Nievera, C., Torgue, J. J., Grimwade, J. E., and Leonard, A. C. (2006). SeqA blocking of DnaA-oriC interactions ensures staged assembly of the E. coli pre-RC. Mol. Cell 24, 581-592. doi: 10.1016/j.molcel.2006.09.016

Niki, H., and Hiraga, S. (1999). Subcellular localization of plasmids containing the oriC region of the Escherichia coli chromosome, with or without the
sopABC partitioning system. Mol. Microbiol. 34, 498-503. doi: 10.1046/j.13652958.1999.01611.x

Noirot-Gros, M. F., Velten, M., Yoshimura, M., McGovern, S., Morimoto, T., Ehrlich, S. D., et al. (2006). Functional dissection of YabA, a negative regulator of DNA replication initiation in Bacillus subtilis. Proc. Natl. Acad. Sci. U.S.A. 103, 2368-2373. doi: 10.1073/pnas.0506914103

Nozaki, S., and Ogawa, T. (2008). Determination of the minimum domain II size of Escherichia coli DnaA protein essential for cell viability. Microbiology 154, 3379-3384. doi: 10.1099/mic.0.2008/019745-0

Ogawa, T., Yamada, Y., Kuroda, T., Kishi, T., and Moriya, S. (2002). The datA locus predominantly contributes to the initiator titration mechanism in the control of replication initiation in Escherichia coli. Mol. Microbiol. 44, 1367-1375. doi: 10.1046/j.1365-2958.2002.02969.x

Ozaki, S., and Katayama, T. (2012). Highly organized DnaA-oriC complexes recruit the single-stranded DNA for replication initiation. Nucleic Acids Res. 40, 1648-1665. doi: 10.1093/nar/gkr832

Ozaki, S., and Katayama, T. (2009). DnaA structure, function, and dynamics in the initiation at the chromosomal origin. Plasmid 62, 71-82. doi: 10.1016/j.plasmid.2009.06.003

Ozaki, S., Noguchi, Y., Hayashi, Y., Miyazaki, E., and Katayama, T. (2012). Differentiation of the DnaA-oriC subcomplex for DNA unwinding in a replication initiation complex. J. Biol. Chem. 287, 37458-37471. doi: 10.1074/jbc.M112.372052

Polaczek, P. (1990). Bending of the origin of replication of E. coli by binding of IHF at a specific site. New Biol. 2, 265-271.

Quon, K. C., Yang, B., Domian, I. J., Shapiro, L., and Marczynski, G. T. (1998). Negative control of bacterial DNA replication by a cell cycle regulatory protein that binds at the chromosome origin. Proc. Natl. Acad. Sci. U.S.A. 95, 120-125. doi: 10.1073/pnas.95.1.120

Rahn-Lee, L., Merrikh, H., Grossman, A. D., and Losick, R. (2011). The sporulation protein SirA inhibits the binding of DnaA to the origin of replication by contacting a patch of clustered amino acids. J. Bacteriol. 193, 1302-1307. doi: 10.1128/JB.01390-10

Rajagopalan, M., Dziedzic, R. A., Zayer, M., Stankowska, D., Ouimet, M. C., Bastedo, D. P., et al. (2010). The Mycobacterium tuberculosis origin of replication and the promoter for immunodominant secreted antigen $85 \mathrm{~B}$ are the targets of MtrA, the essential response regulator. J. Biol. Chem. 285, 15816-15827. doi: 10.1074/jbc.M109.040097

Regev, T., Myers, N., Zarivach, R., and Fishov, I. (2012). Association of the chromosome replication initiator DnaA with the Escherichia coli inner membrane in vivo: quantity and mode of binding. PLOS ONE 7:e36441. doi: 10.1371/journal.pone.0036441

Robinson, A., Brzoska, A. J., Turner, K. M., Withers, R., Harry, E. J., Lewis, P. J., et al. (2010). Essential biological processes of an emerging pathogen: DNA replication, transcription, and cell division in Acinetobacter spp. Microbiol. Mol. Biol. Rev. 74, 273-297. doi: 10.1128/MMBR.00048-09

Roth, A., and Messer, W. (1998). High-affinity binding sites for the initiator protein DnaA on the chromosome of Escherichia coli. Mol. Microbiol. 28, 395-401. doi: 10.1046/j.1365-2958.1998.00813.x

Rozgaja, T. A., Grimwade, J. E., Iqbal, M., Czerwonka, C., Vora, M., and Leonard, A. C. (2011). Two oppositely oriented arrays of low-affinity recognition sites in oriC guide progressive binding of DnaA during Escherichia coli preRC assembly. Mol. Microbiol. 82, 475-488. doi: 10.1111/j.1365-2958.2011. 07827.x

Ryan, V. T., Grimwade, J. E., Camara, J. E., Crooke, E., and Leonard, A. C. (2004). Escherichia coli prereplication complex assembly is regulated by dynamic interplay among Fis, IHF and DnaA. Mol. Microbiol. 51, 1347-1359. doi: 10.1046/j.1365-2958.2003.03906.x

Samitt, C. E., Hansen, F. G., Miller, J. F., and Schaechter, M. (1989). In vivo studies of DnaA binding to the origin of replication of Escherichia coli. EMBO J. 8, 989-993.

Saxena, R., Fingland, N., Patil, D., Sharma, A. K., and Crooke, E. (2013). Crosstalk between DnaA Protein, the initiator of Escherichia coli chromosomal replication, and acidic phospholipids present in bacterial membranes. Int. J. Mol. Sci. 14, 8517-8537. doi: 10.3390/ijms14048517

Saxena, R., Rozgaja, T., Grimwade, J., and Crooke, E. (2011). Remodeling of nucleoprotein complexes is independent of the nucleotide state of a mutant AAA+ protein. J. Biol. Chem. 286, 33770-33777. doi: 10.1074/jbc.M111. 223495 
Schaper, S., and Messer, W. (1995). Interaction of the initiator protein DnaA of Escherichia coli with its DNA target. J. Biol. Chem. 270, 17622-17626. doi: $10.1074 /$ jbc. 270.29 .17622

Schaper, S., Nardmann, J., Lüder, G., Lurz, R., Speck, C., and Messer, W. (2000). Identification of the chromosomal replication origin from Thermus thermophilus and its interaction with the replication initiator DnaA. J. Mol. Biol. 299, 655-665. doi: 10.1006/jmbi.2000.3764

Scholefield, G., Errington, J., and Murray, H. (2012). Soj/ParA stalls DNA replication by inhibiting helix formation of the initiator protein DnaA. EMBO J. 31, 1542-1555. doi: 10.1038/emboj.2012.6

Scholefield, G., and Murray, H. (2013). YabA and DnaD inhibit helix assembly of the DNA replication initiation protein DnaA. Mol. Microbiol. 90, 147-159. doi: $10.1111 / \mathrm{mmi} .12353$

Sekimizu, K., Bramhill, D., and Kornberg, A. (1987). ATP activates dnaA protein in initiating replication of plasmids bearing the origin of the $E$. coli chromosome. Cell 50, 259-265. doi: 10.1016/0092-8674(87)90221-2

Sekimizu, K., Bramhill, D., and Kornberg, A. (1988). Sequential early stages in the in vitro initiation of replication at the origin of the Escherichia coli chromosome. J. Biol. Chem. 263, 7124-7130.

Shaheen, S. M., Ouimet, M. C., and Marczynski, G. T. (2009). Comparative analysis of Caulobacter chromosome replication origins. Microbiology 155, 1215-1225. doi: 10.1099/mic.0.025528-0

Sharan, S. K., Thomason, L. C., Kuznetsov, S. G., and Court, D. L. (2009) Recombineering: a homologous recombination-based method of genetic engineering. Nat. Protoc. 4, 206-223. doi: 10.1038/nprot.2008.227

Simmons, L. A., Felczak, M., and Kaguni, J. M. (2003). DnaA Protein of Escherichia coli: oligomerization at the $E$. coli chromosomal origin is required for initiation and involves specific N-terminal amino acids. Mol. Microbiol. 49, 849-858. doi: 10.1046/j.1365-2958.2003.03603.x

Skarstad, K., Boye, E., and Steen, H. B. (1986). Timing of initiation of chromosome replication in individual Escherichia coli cells. EMBO J. 5, 1711-1717.

Slater, S., Wold, S., Lu, M., Boye, E., Skarstad, K., and Kleckner, N. (1995). E. coli SeqA protein binds oriC in two different methyl-modulated reactions appropriate to its roles in DNA replication initiation and origin sequestration. Cell 82, 927-936. doi: 10.1016/0092-8674(95)90272-4

Smits, W. K., Merrikh, H., Bonilla, C. Y., and Grossman, A. D. (2011). Primosomal proteins DnaD and DnaB are recruited to chromosomal regions bound by DnaA in Bacillus subtilis. J. Bacteriol. 193, 640-648. doi: 10.1128/JB.01253-10

Soufo, C. H., Soufo, H. J., Noirot-Gros, M. F., Steindorf, A., Noirotand, P., and Graumann, P. L. (2008). Cell-cycle-dependent spatial sequestration of the DnaA replication initiator protein in Bacillus subtilis. Dev. Cell 15, 935-941. doi: 10.1016/j.devcel.2008.09.010

Soultanas, P. (2012). Loading mechanisms of ring helicases at replication origins. Mol. Microbiol. 84, 6-16. doi: 10.1111/j.1365-2958.2012.08012.x

Speck, C., and Messer, W. (2001). Mechanism of origin unwinding: sequential binding of DnaA to double- and single-stranded DNA. EMBO J. 20, 1469-1476. doi: 10.1093/emboj/20.6.1469

Speck, C., Weigel, C., and Messer, W. (1997). From footprint to toeprint: a closeup of the DnaA box, the binding site for the bacterial initiator protein DnaA. Nucleic Acids Res. 25, 3242-3247. doi: 10.1093/nar/25.16.3242

Stepankiw, N., Kaidow, A., Boye, E., and Bates, D. (2009). The right half of the Escherichia coli replication origin is not essential for viability, but facilitates multi-forked replication. Mol. Microbiol. 74, 467-479. doi: 10.1111/j.13652958.2009.06877.x

Stougaard, P., Molin, S., and Nordstrom, K. (1981). RNAs involved in copynumber control and incompatibility of plasmid R1. Proc. Natl. Acad. Sci. U.S.A. 78, 6008-6012. doi: 10.1073/pnas.78.10.6008

Taylor, J. A., Ouimet, M. C., Wargachuk, R., and Marczynski, G. T. (2011). The Caulobacter crescentus chromosome replication origin evolved two classes of weak DnaA binding sites. Mol. Microbiol. 82, 312-326. doi: 10.1111/j.13652958.2011.07785.x
Von Freiesleben, U., and Rasmussen, K. V. (1992). The level of supercoiling affects the regulation of DNA replication in Escherichia coli. Res. Microbiol. 143, 655-663. doi: 10.1016/0923-2508(92)90060-2

Von Freiesleben, U., Rasmussen, K. V., Atlung, T., and Hansen, F. G. (2000). Rifampicin-resistant initiation of chromosome replication from oriC in ihf mutants. Mol. Microbiol. 37, 1087-1093. doi: 10.1046/j.1365-2958.2000. 02060.x

Wagner, J. K., Marquis, K. A., and Rudner, D. Z. (2009). SirA enforces diploidy by inhibiting the replication initiator DnaA during spore formation in Bacillus subtilis. Mol. Microbiol. 73, 963-974. doi: 10.1111/j.1365-2958.2009. 06825.x

Weigel, C., Messer, W., Preiss, S., Welzeck, M., Morigen, and Boye, E. (2001). The sequence requirements for a functional Escherichia coli replication origin are different for the chromosome and, a minichromosome. Mol. Microbiol. 40, 498-507. doi: 10.1046/j.1365-2958.2001.02409.x

Weigel, C., Schmidt, A., Rückert, B., Lurz, R., and Messer, W. (1997). DnaA protein binding to individual DnaA boxes in the Escherichia coli replication origin, oriC. EMBO J. 16, 6574-6583. doi: 10.1093/emboj/16.21.6574

Weigel, C., Schmidt, A., Seitz, H., Tüngler, D., Welzeck, M., and Messer, W. (1999). The N-terminus promotes oligomerization of the Escherichia coli initiator protein DnaA. Mol. Microbiol. 34, 53-66. doi: 10.1046/j.13652958.1999.01568.x

Wolanski, M., Donczew, R., Zawilak-Pawlik, A., and Zakrzewska-Czerwinska, J. (2014). oriC-encoded instructions for the initiation of bacterial chromosome replication. Front. Microbiol. 5:735. doi: 10.3389/fmicb.2014.00735

Wolanski, M., Jakimowicz, D., and Zakrzewska-Czerwinska, J. (2012). AdpA, key regulator for morphological differentiation regulates bacterial chromosome replication. Open Biol. 2, 120097. doi: 10.1098/rsob.120097

Yoshida, Y., Obita, T., Kokusho, Y., Ohmura, T., Katayama, T., Ueda, T., et al. (2003). Identification of the region in Escherichia coli DnaA protein required for specific recognition of the DnaA box. Cell Mol. Life Sci. 60, 1998-2008. doi: 10.1007/s00018-003-3176-7

Yung, B. Y., Crooke, E., and Kornberg, A. (1990). Fate of the DnaA initiator protein in replication at the origin of the Escherichia coli chromosome in vitro. J. Biol. Chem. 265, 1282-1285.

Zawilak-Pawlik, A., Donczew, R., Szafranski, S., Mackiewicz, P., Terradot, L., and Zakrzewska-Czerwinska, J. (2011). DiaA/HobA and DnaA: a pair of proteins co-evolved to cooperate during bacterial orisome assembly. J. Mol. Biol. 408, 238-251. doi: 10.1016/j.jmb.2011.02.045

Zawilak-Pawlik, A., Kois, A., Majka, J., Jakimowicz, D., Smulczyk-Krawczyszyn, A., Messer, W., et al. (2005). Architecture of bacterial replication initiation complexes: orisomes from four unrelated bacteria. Biochem. J. 389, 471-481. doi: 10.1042/BJ20050143

Zawilak-Pawlik, A., Kois, A., Stingl, K., Boneca, I. G., Skrobuk, P., Piotr, J., et al. (2007). HobA-a novel protein involved in initiation of chromosomal replication in Helicobacter pylori. Mol. Microbiol. 65, 979-994. doi: 10.1111/j.1365-2958.2007.05853.x

Zorman, S., Seitz, H., Sclavi, B., and Strick, T. R. (2012). Topological characterization of the DnaA-oriC complex using single-molecule nanomanipuation. Nucleic Acids Res. 40, 7375-7383. doi: 10.1093/nar/gks371

Conflict of Interest Statement: The authors declare that the research was conducted in the absence of any commercial or financial relationships that could be construed as a potential conflict of interest.

Copyright (c) 2015 Leonard and Grimwade. This is an open-access article distributed under the terms of the Creative Commons Attribution License (CC BY). The use, distribution or reproduction in other forums is permitted, provided the original author(s) or licensor are credited and that the original publication in this journal is cited, in accordance with accepted academic practice. No use, distribution or reproduction is permitted which does not comply with these terms. 